\title{
Synthesis of carbon nanotubes using microwave oven
}

\begin{abstract}
Advances in the synthesis of carbon nanotubes (CNTs) have emerged as a result of the properties and potential application of carbon nanotubes. We demonstrated a simple approach of using domestic microwave oven with $600 \mathrm{~W}$ at $2.45 \mathrm{GHz}$ which was modified to produce CNTs from a carbon source on coated silicon oxide substrate. The Raman spectroscopy showed the graphitic nature of the obtained CNTs, with intensity ratio ID/IG calculated to be 0.92. Field emission scanning electron microscope (FESEM) reveals CNTs are produced on the substrate surface with outer diameter range of 11-44 nm and length of about $0.25 \mathrm{\varepsilon m}$. HRTEM further confirmed the graphitic nature of the CNTs obtained. The purity of the nanotubes was analyzed with energy dispersive X-ray (EDX) which showed atomic weight of 98\% carbon purity. This paper shows that domestic microwave oven can be used to synthesize CNTs with polymer as the carbon source via plasma catalytic decomposition which was found to be fast, economical and clean technique.
\end{abstract}

Keyword: Carbon nanotubes; Microwave oven; Plasma; Polyethylene; Spectroscopy 